\title{
LIPAEMIA RETINALIS: A QUESTION OF CHYLOMICRONS?
}

\author{
SANDRA RAYNER ${ }^{1}$, NICHOLAS LEE $^{2}$, DAVID LESLIE $^{3}$ and GRAHAM THOMPSON ${ }^{4}$
}

\begin{abstract}
SUMMARY
Lipaemia retinalis is a rarely described condition which occurs in certain types of both primary and secondary hyperlipidaemia. It presents with a striking fundal appearance. We report 7 cases of lipaemia retinalis and ocular manifestations in hyperlipidaemia which presented over a 12 year period to our hospitals. These illustrate the different presentations and stages of the disease, and other fundal abnormalities seen in hyperlipidaemia.
\end{abstract}

Lipaemia retinalis, which was first described in 1880 by Heyl ${ }^{1}$, occurs in certain types of both primary and secondary hyperlipidaemia. The principal fundal abnormality in lipaemia retinalis is a milky discolouration of retinal vessels. This can vary in degree from barely detectable peripheral vessel changes to cream colouration of all retinal vessels with a 'salmon'-coloured fundus. The grading system used to describe the stages of the disease is given in Table I. The incidence of lipaemia retinalis correlates approximately with the level of plasma triglycerides. However, the relationship is indirect because the key factor is the level of chylomicrons, the large lipoproteins which serve to transport triglycerides. To a lesser extent slightly smaller VLDL (very low density lipoprotein) macromolecules are also involved. The fundal appearance is believed to be caused by the visualisation of increased levels of chylomicrons in the circulating retinal blood. However, not all patients with even grossly elevated chylomicron or triglyceride levels show evidence of lipaemia retinalis, demonstrating that other factors, such as haematocrit and difference in translucency of the retinal and choroidal vessels, are important. ${ }^{2}$

Lipaemia retinalis has been described in both

From: ${ }^{1}$ Hammersmith Hospital; ${ }^{2}$ Western Eye Hospital; ${ }^{3}$ St Bartholomew's Hospital; ${ }^{4}$ St George's Hospital, London, UK. Correspondence to: S.A. Rayner, FRCOphth, Immunology Department, Hammersmith Hospital, Du Cane Road, London W12 0NN, UK. primary and secondary hyperlipidaemias. With regard to primary hyperlipidaemias, it is seen only in those with elevated chylomicron levels, i.e. types I, III, IV and V (Table II). ${ }^{3,4}$ In the secondary hyperlipidaemias, a major cause of lipaemia retinalis is chylomicronaemia resulting from uncontrolled diabetes mellitus. ${ }^{5-8}$

\section{CASE REPORTS}

We present 7 patients: 2 with diabetes causing secondary hyperlipidaemia; 2 with a primary hyperlipidaemia associated with concurrent diabetes; 2 with primary hyperlipidaemia unassociated with diabetes and 1 with a short-term hyperlipidaemia caused by impairment of lipid metabolism during a viral illness (Table III). Lipaemia is often a transient condition, not infrequently lasting only a few hours. For this reason in several of the case reports presented few details were recorded in the patients' notes and the summaries given below are therefore necessarily brief.

\section{Transient Lipaemia Retinalis}

Case 1. This 30-year-old non-insulin-dependent diabetic woman had grossly elevated triglyceride and cholesterol levels on presentation (cholesterol $24 \mathrm{mmol} / \mathrm{l}$, triglycerides $104 \mathrm{mmol} / \mathrm{l})$. On correction of her uncontrolled diabetes and lowering of the lipid levels the grade I-II lipaemia retinalis rapidly resolved.

Case 2. A 29-year-old woman was admitted with uncontrolled insulin-dependent diabetes mellitus. Again early lipaemia retinalis was visible on fundos-

Table I. Grading system to describe the stages of lipaemia retinalis

\begin{tabular}{lll}
\hline Grade I & Early & $\begin{array}{l}\text { Peripheral vessels have a creamy tint } \\
\text { Grade II }\end{array}$ \\
Moderate & $\begin{array}{l}\text { Extension of cream-coloured vessels } \\
\text { towards the disc }\end{array}$ \\
Grade III & Marked & $\begin{array}{l}\text { All vessels are cream-coloured, arteries } \\
\text { and veins indistinguishable. Fundus } \\
\text { appears a salmon colour }\end{array}$ \\
\hline
\end{tabular}


Table II. The widely used WHO classification provides a shorthand for describing the lipoprotein profile in hyperlipidaemia but does not indicate the underlying cause

\begin{tabular}{|c|c|c|c|}
\hline \multirow{2}{*}{$\begin{array}{l}\text { Lipoprotein } \\
\text { pattern }\end{array}$} & \multicolumn{2}{|c|}{ Major elevation in plasma } & \multirow[b]{2}{*}{ Ocular features } \\
\hline & Lipoprotein & Triglycerides & \\
\hline Type I & Chylomicrons & Triglycerides & $\begin{array}{l}\text { Lipaemia retinalis; iris and retinal xanthomas; lipid keratopathy; adult- } \\
\text { onset Coats' disease }\end{array}$ \\
\hline Type IIa and b & LDL/VLDL & $\begin{array}{l}\text { Cholesterol and } \\
\text { triglycerides }\end{array}$ & $\begin{array}{l}\text { Xanthelasma; corneal arcus in third to fifth decades; xanthomas of retina, } \\
\text { choroid or conjunctiva with lipid keratopathy }\end{array}$ \\
\hline Type III & Remnants & $\begin{array}{l}\text { Triglycerides and } \\
\text { cholesterol }\end{array}$ & Early onset of arcus senilis; rarely lipaemia retinalis; xanthomas \\
\hline Type IV & VLDL & Triglycerides & Lipaemia retinalis; xanthomas \\
\hline Type V & $\begin{array}{l}\text { VLDL and } \\
\text { chylomicrons }\end{array}$ & $\begin{array}{l}\text { Triglycerides and } \\
\text { cholesterol }\end{array}$ & Lipaemia retinalis; xanthomas not uncommon \\
\hline
\end{tabular}

Table III. Summary table of the lipid values of the patients presenting with lipaemia retinalis

\begin{tabular}{|c|c|c|c|c|c|c|}
\hline \multirow[b]{2}{*}{ Case } & \multirow[b]{2}{*}{ Sex } & \multirow[b]{2}{*}{$\begin{array}{c}\text { Age } \\
\text { (years) }\end{array}$} & \multicolumn{2}{|c|}{ Blood lipid levels } & \multirow[b]{2}{*}{$\begin{array}{l}\text { Type of } \\
\text { diabetes }\end{array}$} & \multirow[b]{2}{*}{$\begin{array}{l}\text { Primary or secondary } \\
\text { hyperlipidaemia }\end{array}$} \\
\hline & & & $\begin{array}{l}\text { Cholesterol } \\
(\mathrm{mmol} / \mathrm{l})\end{array}$ & $\begin{array}{l}\text { Triglycerides } \\
\text { (mmol/l) }\end{array}$ & & \\
\hline 1 & $\mathrm{~F}$ & 30 & 24 & 104 & NIDDM & Secondary \\
\hline 2 & $\mathrm{~F}$ & 29 & $\mathrm{nr}$ & $\mathrm{nr}$ & IDDM & Secondary \\
\hline 3 & $\mathrm{M}$ & 57 & 55 & 123 & NIDDM & Primary and secondary \\
\hline 4 & $\mathrm{M}$ & 44 & 38.7 & 139 & No DM & Secondary \\
\hline 5 & $\mathrm{M}$ & 40 & $>30$ & $>30$ & No DM & Primary \\
\hline 6 & $\mathrm{~F}$ & 60 & 45 & 70 & IDDM & Primary \\
\hline 7 & M & 48 & $>30$ & $>30$ & No DM & Primary \\
\hline
\end{tabular}

DM, diabetes mellitus; IDDM, insulin-dependent DM; NIDDM, non-insulin-dependent DM; nr, not recorded.

Normal range: cholesterol, $3.50-6.50 \mathrm{mmol} / \mathrm{l}$; triglycerides $0.35-2.10 \mathrm{mmol} / \mathrm{l}$.

copy initially, but after administration of insulin and correction of the metabolic disturbance this cleared within 1 hour. Lipid levels were not recorded during the acute stages.

\section{Type 3 Lipaemia Retinalis}

Case 3. A 57-year-old man presented with a 1 week history of blurred and double vision. Visual acuities were $6 / 9$ in either eye. Nine months previously he had been diagnosed as a non-insulin-dependent diabetic, but his compliance with treatment had been poor (initial $\mathrm{Hb}_{\mathrm{A} 1 \mathrm{c}}=16 \%$, normal range 4.5 $8.5 \%)$. At the time of presentation to the eye department he had systemic symptoms of thirst and nocturia. There was a strong family history of both

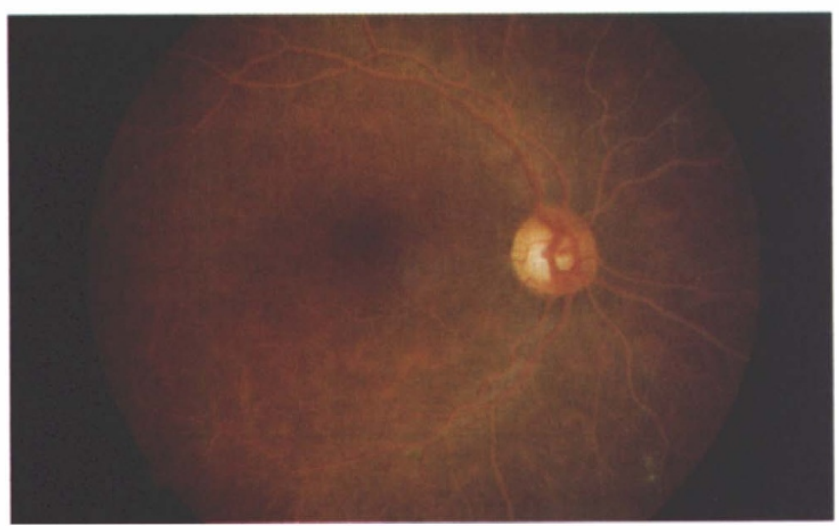

(a) heart disease and diabetes. At presentation his fundus showed a salmon-coloured appearance, with cream-coloured vessels demonstrating grade III lipaemia retinalis (Fig. 1a, b). There were very few other retinal diabetic changes. Lipid profiles showed a type $\mathrm{V}$ hyperlipidaemia and his serum at presentation was grossly lipaemic, showing a densely opalescent serum with a chylomicron supernatant (Fig. 2). $\mathrm{He}$ was treated with a regular regime of insulin and a low-fat diet. The lipaemia retinalis resolved over 6 weeks (Fig. 3a, b). However, despite good compliance with therapy, 3 months later his lipid profile remained abnormal (cholesterol $14.8 \mathrm{mmol} / \mathrm{l}$, triglycerides $20.3 \mathrm{~mm} / \mathrm{l}$ ). Familial lipoprotein lipase deficiency was confirmed and he was started on

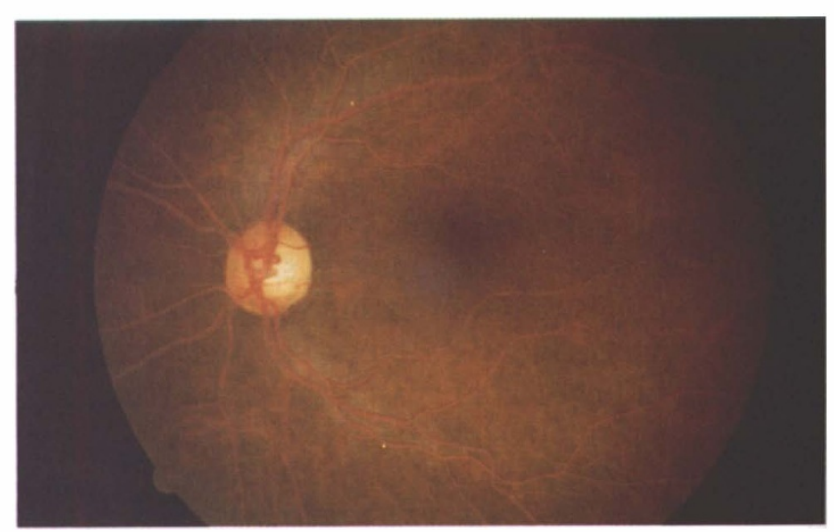

(b)

Fig. 1. Case 3. Fundus photograph showing lipaemia retinalis stage 3 in the right eye (a) and left eye (b). The arteries and veins appear a similar pale colour, distinguishable only by their size. With the choroidal vessels having the same pallor the fundus appears salmon in colour. 


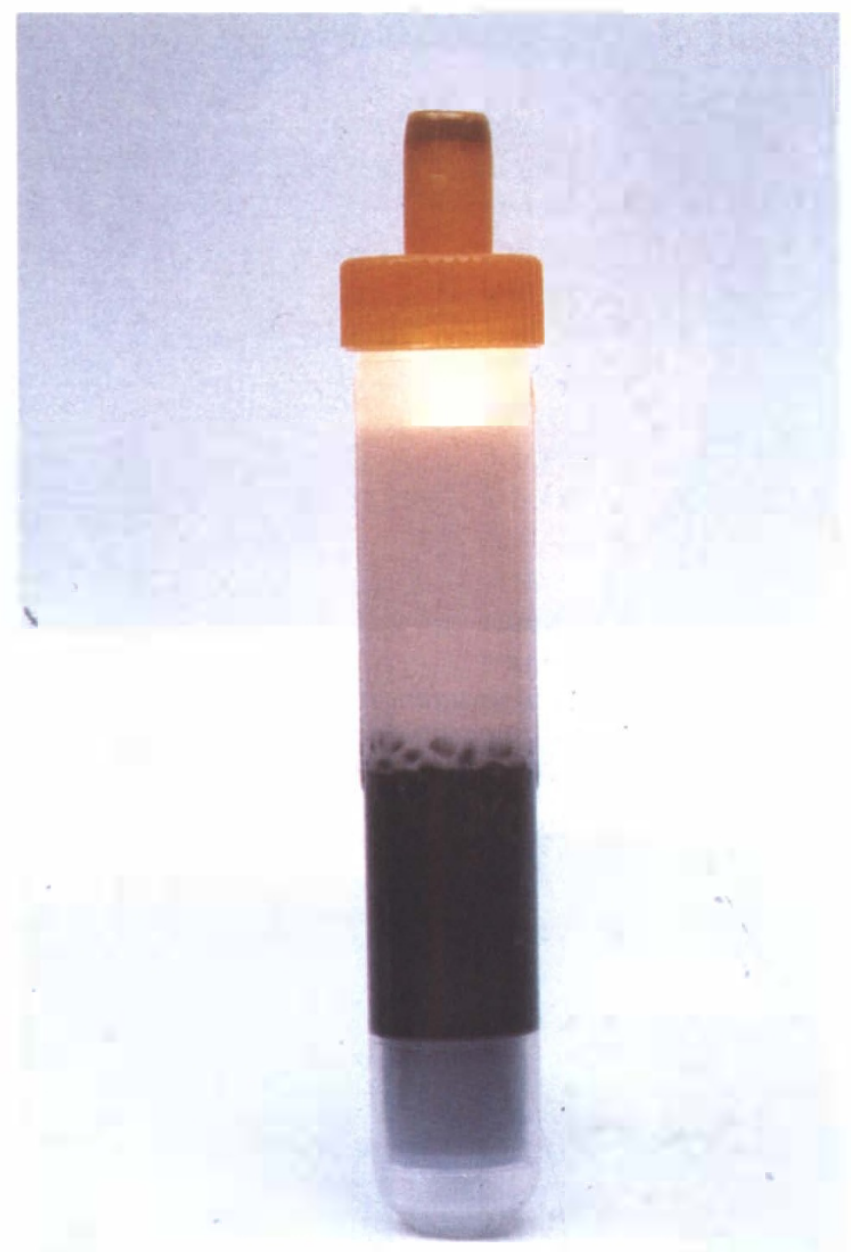

Fig. 2. Case 3. The acute blood sample after allowing the blood to stand for 24 hours. The serum is opalescent from grossly elevated VLDL. There is a thin supernatant of chylomicrons.

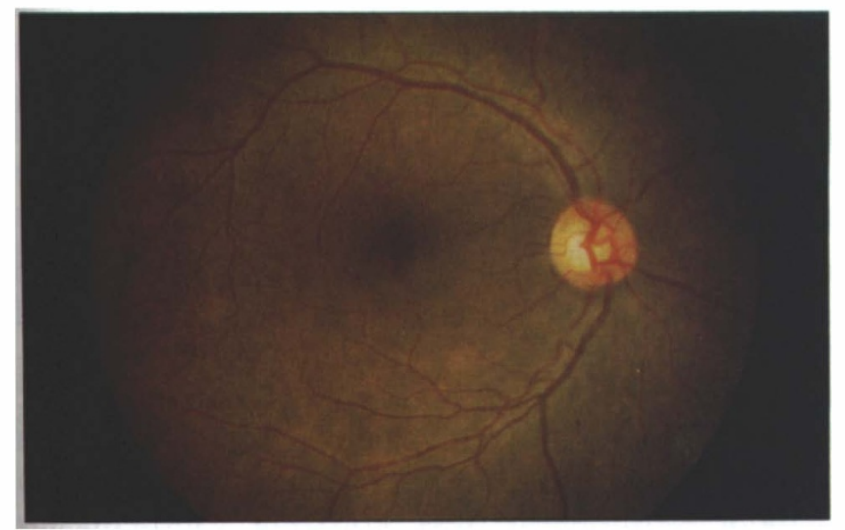

(a)

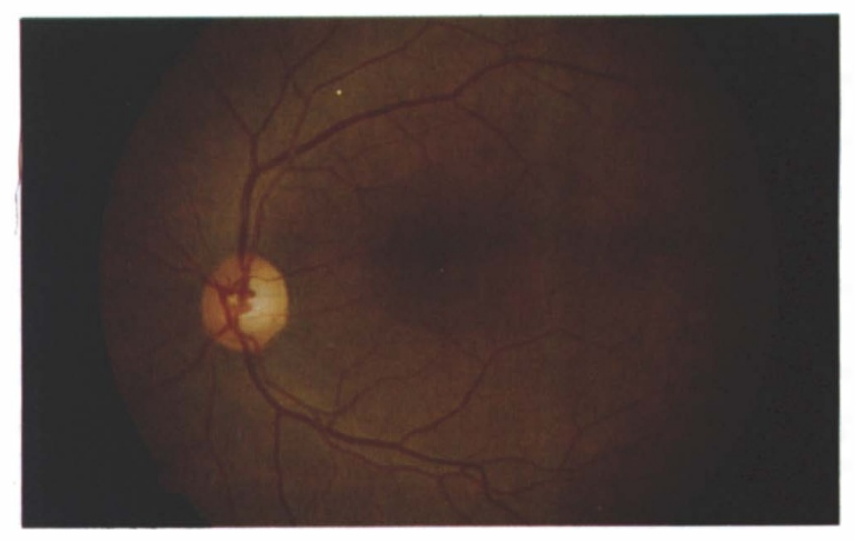

(b)

Fig. 3. Case 3. Fundus photographs of the right eye (a) and left eye (b) after 6 weeks with a controlled diabetic status. Although the lipids remain elevated above normal they are not high enough to cause the blood to be lipaemic and the fundus has returned to a normal appearance. 


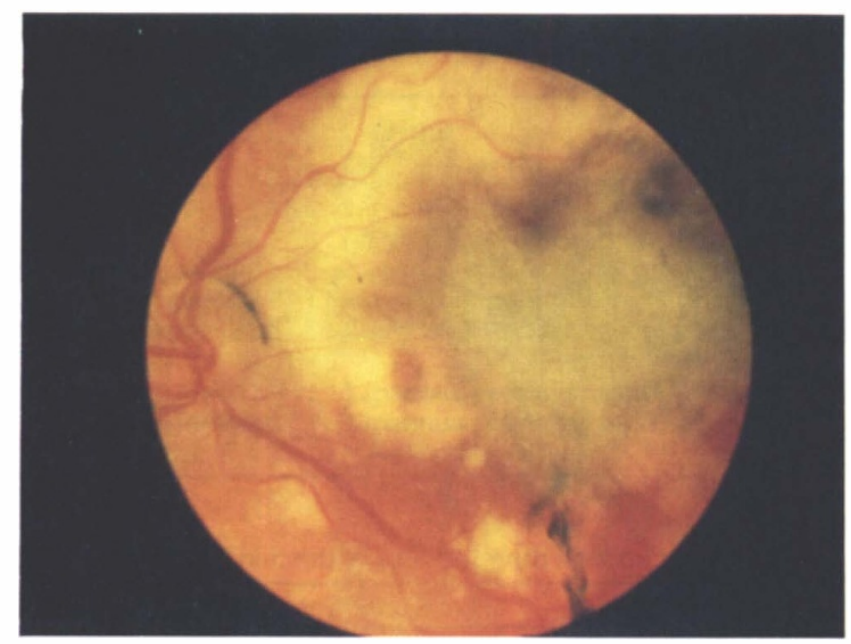

Fig. 4. Case 6. Lipid exudation or xanthelasma of the retina associated with hyperlipidaemia. These appearances did not resolve with control of the hyperlipidaemia.

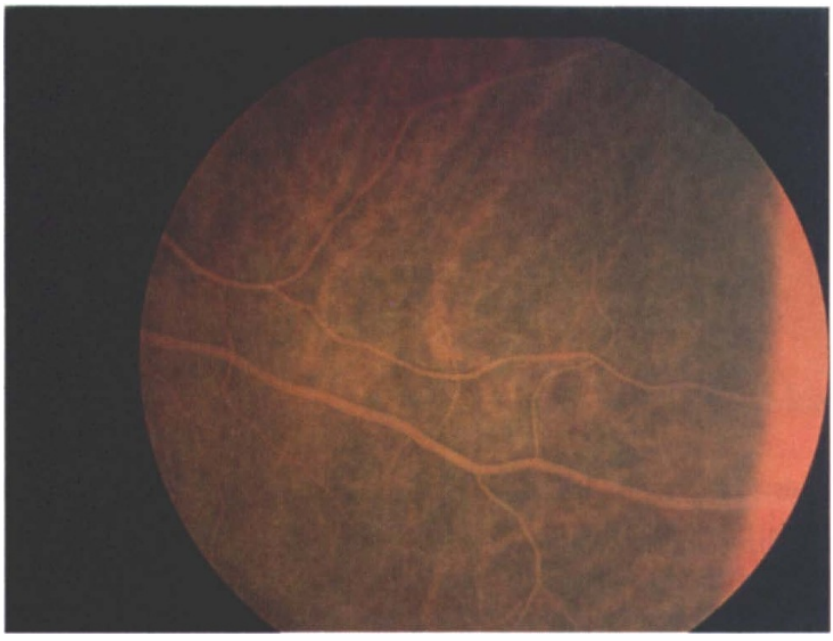

(a)

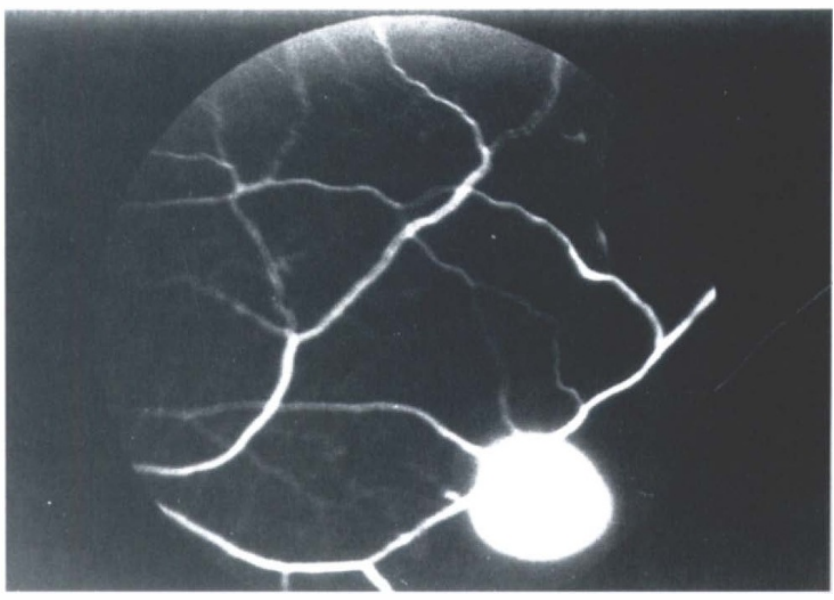

(b)

Fig. 5. Case 7. (a) Peripheral retinal image. (b) Peripheral fluorescein angiogram. These show peripheral non-perfusion due to infarction from hyperviscosity associated with the hyperlipidaemia.

treatment of a fibric acid derivative to control his hyperlipidaemia.

Case 4. A 44-year-old non-diabetic man developed hyperlipidaemia with typical lipaemia retinalis during a viral illness (cholesterol $38.7 \mathrm{mmol} / \mathrm{l}$, triglycerides $139 \mathrm{mmol} / \mathrm{l})$. The ophthalmoscopic appearance settled on resolution of the viral illness, which was presumed to have caused an impairment of lipid metabolism. His blood lipid values returned to normal on recovery. No underlying hyperlipidaemia was subsequently found.

Case 5. A 40-year-old man, the son of case 6, was tested for hyperlipidaemia and found to have raised lipid levels (cholesterol $>30 \mathrm{mmol} / \mathrm{l}$, triglycerides $>30$ $\mathrm{mmol} / \mathrm{l})$. He had no visual symptoms and was not diabetic. On fundal examination at presentation a typical lipaemia retinalis picture was documented.

\section{Massive Lipid Exudation}

Case 6. A 60-year-old woman developed mild diabetes following a course of steroids for suspected temporal arteritis. The diabetes was initially controlled by diet and oral hypoglycaemic agents. Fundal examination revealed a lipid exudation around the supero- and inferotemporal arcades. No diabetic retinopathy was apparent. Subsequently there was a massive lipid exudation into the central macular region (Fig. 4). At the same time xanthelasmata appeared on the eyelids and lipomata on the elbows. Serum lipids were found to be markedly abnormal (cholesterol $45 \mathrm{mmol} / \mathrm{l}$, triglycerides 70 
mmol/l). Her treatment was changed to regular insulin injections, a low-fat diet and bezafibrate. This controlled her hyperlipidaemia but her vision did not improve beyond 'counting fingers' for the right eye and $6 / 12$ for the left.

\section{Peripheral Vascular Closure}

Case 7. On investigation for headaches and dizziness a 48-year-old alcoholic man was found to have type $\mathrm{V}$ hyperlipidaemia (cholesterol $>30 \mathrm{mmol} / \mathrm{l}$, triglycerides $>30 \mathrm{mmol} / \mathrm{l}$ ). His symptoms were ascribed to cerebral hyperviscosity possibly associated with the hyperlipidaemia. Vision was normal. As well as the typical lipaemia retinalis appearance of his fundus he developed peripheral vascular closure confirmed on fluorescein angiography (Fig. 5). He underwent plasmapheresis to ensure rapid reduction of his hyperlipidaemia. Further control was achieved by reduction of his alcohol intake and a low-fat diet. This corrected his hyperlipidaemia and the lipaemia retinalis cleared, but the peripheral vascular closure remained.

\section{DISCUSSION}

These patients demonstrate some of the varied clinical presentations of lipaemia retinalis, both classic and with associated pathology. Lipaemia retinalis is a condition usually described as 'rare', but in 1982 Brunzell and Bierman ${ }^{4}$ described it as occurring in $23 \%$ of patients with 'chylomicronaemia' (i.e. with triglyceride $>22.5 \mathrm{mmol} / \mathrm{l}$ ).

The first appearances of lipaemia retinalis can be seen in the peripheral parts of the retina. The vessels take on a creamy or pink appearance making it difficult to distinguish between the veins and arteries. This process gradually increases and spreads towards the disc. Similar changes in the choroidal vessels cause the fundal background to take on a salmon colour. Long-standing lipaemia retinalis may lead to lipid exudation or xanthoma-like lesions which are initially situated close to the vessels. Vision is initially normal, but may become reduced by more advanced lipaemia. Once the hyperlipidaemia has been treated the clinical picture of lipaemia retinalis quickly resolves. However, persistent lipaemia retinalis can lead to massive irreversible lipid exudation with loss of vision.

The pathological appearance of lipaemia retinalis is believed to be due to visualisation of lactescent serum flowing through retinal and choroidal vessels

Table IV. Diameter of plasma lipoproteins

\begin{tabular}{lc}
\hline Plasma lipoprotein & Mean diameter $(\mathrm{nm})$ \\
\hline Chylomicron & $100-1000$ \\
VLDL & 34 \\
IDL & 27 \\
LDL & 22 \\
HDL & $5-12$ \\
\hline
\end{tabular}

of a hyperlipidaemic patient. The cause of lactescent serum was discussed in 1949 by Ahrens and Kunkel. ${ }^{9}$ They postulated that since particles in suspension need to be a certain size in order to scatter light and produce a turbid appearance, lipaemic sera must contain particles of at least 0.25 wavelength of visible light, i.e. about $100 \mathrm{~nm}$.

It has since been shown that there are at least five classes of lipid transport molecule or lipoprotein. These can be separated by size using preparative ultracentrifugation (Table IV). Chylomicrons are the largest lipoprotein macromolecules. They range in size from 100 to $1000 \mathrm{~nm}$. VLDL are generally smaller but some reach a dimension of $100 \mathrm{~nm}$. The smaller lipoproteins play an important role in fat transport and metabolism but do not contribute to the visible turbidity of serum. If a sample of hyperlipidaemic serum is allowed to stand at $4{ }^{\circ} \mathrm{C}$, chylomicrons form a hazy surface layer (Fig. 2). Smaller lipoprotein molecules do not scatter light and are thus transparent. Likewise circulating blood with a high level of chylomicrons can give the appearance of lipaemia retinalis in the retinal and choroidal vessels.

Chylomicrons are the lipoprotein form in which most of the dietary triglyceride is transported from its intestinal site of absorption to the systemic circulation. They are normally rapidly removed from plasma and should be undetectable after a 12 hour fast. It is likely that it is the high proportion of triglycerides in chylomicrons which leads to the observed association of raised triglyceride levels and lipaemia retinalis. However, it is thought to be the light-scattering effect of chylomicrons which causes the clinical picture in the fundi.

Cases 6 and 7 illustrate additional retinal pathology seen in hyperlipidaemia, although these cases did not have classic lipaemia retinalis. Kurz et al. ${ }^{10}$ also observed massive lipid extravasation with visual loss and described the histological changes at postmortem. A second case they describe showed progressive obstruction of vessels with lipid causing visual field deficit.

\section{CONCLUSION}

Lipaemia retinalis is an initially asymptomatic condition which can be demonstrated in some cases of hyperchylomicronaemia. It may resolve rapidly on the institution of lipid-lowering measures and particularly quickly on the correction with insulin of hyperlipidaemia secondary to diabetes. Late complications such as massive lipid exudation may cause visual deficit which is at times irreversible.

Because it does not normally cause visual symptoms and presents first in the peripheral retina, it is possible that lipaemia retinalis is an underdiagnosed condition. Physicians might find that a close inspec- 
tion of the peripheral retina at diagnosis of hyperlipidaemia reveals a higher incidence. Lipaemia retinalis is a good clinical indicator with high specificity for high chylomicron and triglyceride levels, and as such should not be ignored as a simple diagnostic sign. If hyperlipidaemia persists, lipidlowering measures should be instituted to prevent cardiovascular disease. The risk to vision of prolonged hyperlipidaemia cannot be ignored.

Key words: Lipaemia retinalis, Hyperlipidaemia, Chylomicrons.

\section{REFERENCES}

1. Heyl AG. Intraocular lipaemia. Trans Am Ophthalmol Soc 1880;3:55.

2. Vinger PF, Sachs BA. Ocular manifestations of hyperlipoproteinaemia. Am J Ophthalmol 1970;70: 563-72.
3. Thomas PK, Smith EB. Ocular manifestations in idiopathic hyperlipidaemia and xanthomatosis. $\mathrm{Br} \mathrm{J}$ Ophthalmol 1958;42:501-6.

4. Brunzell JD, Bierman EL. Chylomicronaemia syndrome: interaction of genetic and acquired hypertriglyceridaemia. Med Clin North Am 1982;66:455-68.

5. Chait A, et al. Chylomicronaemia syndrome in diabetes mellitus. Diabetes Care 1981;4:343-8.

6. Chase LA. Diabetic lipaemia retinalis. Can Med Assoc J 1927;17:197-204.

7. Martinez KR, et al. Lipaemia retinalis (photo essay). Arch Ophthalmol 1992;110:1171.

8. Bagdade JD, et al. Diabetic lipaemia. N Engl J Med 1967;276:427-33.

9. Ahrens EH, Kunkel HG. The stabilisation of serum lipid emulsions by serum phospholipids. J Exp Med 1949;90:409.

10. Kurz GH, et al. The retina in type V hyperlipoproteinaemia. Am J Ophthalmol 1976;82:32-43. 